\title{
A double-blind randomized controlled trial of topical Curcuma xanthorrhiza Roxb. on mild psoriasis: clinical manifestations, histopathological features, and K6 expressions
}

\author{
Githa Rahmayunita, ${ }^{1,2}$ Tjut N.A. Jacoeb, ${ }^{1,2}$ Endi Novianto, ${ }^{1,2}$ Wresti Indriatmi, ${ }^{1}$ Rahadi Rihatmadja, ${ }^{1,2}$ \\ Erdina H.D. Pusponegoro ${ }^{1,2}$ \\ ${ }^{1}$ Department of Dermatology and Venereology, Faculty of Medicine, Universitas Indonesia, Cipto Mangunkusumo Hospital, \\ Jakarta, Indonesia \\ ${ }^{2}$ Indonesian Psoriasis Study Group, Indonesian Society of Dermatology and Venereology, Jakarta, Indonesia
}

\section{ABSTRACT}

Background: Curcuma xanthorrhiza Roxb. exerts its anti-inflammatory effects by reducing the concentration of IL-6, IL-8, and phosphorylase kinase, which has role in keratinocyte proliferation. Our study aimed to evaluate the efficacy of $C$. xanthorrhiza in psoriasis.

Methods: From 18 to 59 year-old patients with mild psoriasis, 2 similar lesions were selected. The severity assessment was based on the psoriasis area severity index (PASI), Trozak score, and K6 expression. Using a double-blinded randomized method, lesion was treated with $1 \%$ C. xanthorrhiza ointment vs placebo for 4 weeks. The results were analyzed by the chi-square test using STATA ${ }^{\mathrm{TM}}$ V.12 software (Stata Corp.).

Results: The study was conducted in 2010 to 2012 with 17 subjects participated. The median of PASI score were reduced significantly in both lesions, either treated with $1 \%$ C. xanthorrhiza ointment vs placebo; however when compared between the group, it was not significant ( $\mathrm{p}=0.520$ ). The Trozak score were reduced in lesions treated with $1 \%$ C. xanthorrhiza ointment; but it was not significant $(p=0.306)$. In lesions treated with placebo, the Trozak score was increased significantly. The difference of Trozak score between lesions treated with $C$. xanthorrhiza and placebo was significant $(\mathrm{p}=0.024)$. There was no significant difference of $\mathrm{K} 6$ expression in lesions treated with $1 \%$ C. xanthorrhiza ointments or placebo as well as on the difference of mean values of K6 expression between the group $(\mathrm{p}=0.827)$.

Conclusion: Based on the results, $1 \%$ C. xanthorrhiza ointment is effective treatment option for mild psoriasis, but longer followup period is suggested to confirm this results. C. xanthorrhiza ointment is safe for topical administration as there were no side effects reported in this study.

Keywords: topical Curcuma xanthorrhiza, psoriasis, PASI, Trozak score, K6 expressions

pISSN: 0853-1773 • eISSN: 2252-8083 • https://doi.org/10.13181/mji.v27i3.2511 • Med J Indones. 2018;27:178-84

- Received 20 Dec 2017• Accepted 20 Apr 2018

Corresponding author: Githa Rahmayunita

githa_rahmayunita@yahoo.com

Copyright @ 2018 Authors. This is an open access article distributed under the terms of the Creative Commons Attribution-NonCommercial 4.0 International License (http://creativecommons.org/licenses/by-nc/4.0/), which permits unrestricted non-commercial use, distribution, and reproduction in any medium, provided the original author and source are properly cited. 
Psoriasis is a chronic and recurrent inflammation of the skin. The disease can also affect mucous membranes, nails, and joints. The typical clinical manifestation is erythematous patches with transparent mica-like scales and the sites of predilection include knees, elbows, scalp, retroauricular, and lumbar regions. ${ }^{1}$ The etiopathogenesis of psoriasis is complex, which includes genetic factors, immunological disorders with autoimmune processes and apoptosis failure. ${ }^{1,2-4}$ In general, psoriasis is divided into three categories, i.e., mild, moderate, and severe. The most common form of psoriasis is plaque type psoriasis, also known as psoriasis vulgaris. The management of psoriasis depends on its severity. Moderate and severe psoriasis are treated with systemic medications such as methotrexate, acitretin, and cyclosporin. ${ }^{1}$ Furthermore, phototherapy using ultraviolet (UV) A and UVB radiation is also commonly administered. Recently, biological agents have been commonly used. Topical treatment is indicated for mild psoriasis. Common topical treatments include corticosteroids, vitamin D3, vitamin A derivatives, and tar preparations. ${ }^{5}$ Nevertheless, those medications have side effects that should be warned, particularly in long-term use. ${ }^{2,5}$ Therefore, effective and safe treatment with affordable price is still being searched. ${ }^{6}$

Curcuma xanthorrhiza Roxb. or Javanese Turmeric-one of an Indonesian herbal medicine, is a member of the ginger family (Zingiberaceae)., ${ }^{7,8}$ Curcuma xanthorrhiza in which is the utilized part is rhizome, may have effects in preventing and treating various diseases because of its effects as anti-inflammatory, lowering cholesterol levels, diuretics, increasing breast milk production and as a remedy for joint pain. ${ }^{9,10} \mathrm{~A}$ study on human keratinocytes (HaCaT line) treated with extracts of Curcuma longa rhizomes, demonstrates its effect on a reduced activity of pro-inflammatory cytokines of IL-6 and IL-8, which is equal to topical betamethasone 17 -valerate. ${ }^{10}$ Curcuma xanthorrhiza has shown some significant effects on psoriasis related on several receptors to which curcumin binds, which are 5-lipoxygenase (5LOX), xanthine oxidase, thioredoxin reductase, cyclooxygenase-2 (COX-2), p-glycoprotein, glutathione S-transferase (GST), protein kinase A (PKA), protein kinase C (PKC), cPK, PK, Ca2+-dependent protein kinase (CDPK), and glutathione. ${ }^{11}$ Heng et $\mathrm{al}^{12}$ administered topical curcumin for psoriasis patients compared to calcipotriol. The study showed that curcumin can lower the activity of phosphorylase kinase enzyme more than calcipotriol. The decrease in phosphorylase kinase activity in curcumin and calcipotriol-treated psoriasis was associated with a decrease in keratinocyte transferrin receptor expression and with decrease in the severity of parakeratosis and the density of epidermal CD8+ T cells. Our study was aimed to identify the effectivity of $C$. xanthorrhiza on psoriasis.

\section{METHODS}

The study evaluated patients with mild psoriasis in the Dermatovenereology Outpatient Unit at Cipto Mangunkusumo Hospital Jakarta between 2010 and 2012. In each of patients, 2 lesions with similar severity were determined. One lesion was treated with $1 \%$ C. xanthorrhiza ointment; while the other lesion was treated with placebo containing white vaseline album (vehicle of the C. xanthorrhiza ointment) with similar matching and packaging, which prepared by PT Soho Industri Pharmasi. Both treatments were given for 4 weeks experimentally in a doubleblind randomized controlled trial. The $1 \% C$. xanthorrhiza and placebo ointments were coded by other investigator which either investigators or the patients did not know.

Patients were included in our study when they were aged 18 to 59 years old with mild psoriasis, were not pregnant or breastfeeding, not having topical treatment for psoriasis in the last 2 weeks and were not having systemic or phototherapy treatment for psoriasis in the last 4 weeks. The number of subjects taken consecutively was 20 subjects. Prior to the study, written consent had been asked and the study had received ethics approval from the Ethic Committee of Health Studies at Faculty of Medicine, Universitas Indonesia-Cipto Mangunkusumo Hospital (292/ pT02.FK/43/N/2010).

In the beginning of the study, evaluation of psoriasis severity was conducted clinically based on the degree of erythematous (E) and induration of plaque thickness (I). Both signs characterized the presence of inflammation; while the thickness of scales (S) indicated excessive keratinocytes production. The lesions were evaluated using 
psoriasis area severity index (PASI), i.e. by summing the degree of erythema severity (1-4), infiltration (1-4) and scales (1-4), which were multiplied with the precentage of affected body surface area. In this study we only calculated the size of the lesions treated with $1 \%$ C. xanthorrhiza ointment and placebo as the affected body surface area (Figure 1).

Moreover, biopsy and histopathological examination stained with hematoxylin eosin (HE) were also conducted to calculate Trozak score and immunohistochemical staining was carried out to calculate the percentage of K6 expression. The calculation of Trozak score was performed at the Dermatopathology Division, Department of Dermatology Venereology Cipto Mangunkusumo Hospital. Features in histopathological finding is assigned using value of 1,2 , and 3 as follows: regular elongation of the rete ridges (1), club shaped rete ridges (2), elongation and edema of dermal papillae (1), perivascular mononuclear infiltrate in the upper dermis of papillae (1), absence of granular layer (focal:1, total:2), parakeratosis (focal:1, total:2), suprapapillary plate thinning (2), mitosis above basal cell layer (2), Munro microabscess (3), spongiformis pustule (3) (Figure 2A). ${ }^{13}$ The presentation of K6 expression was evaluated at the Department of Anatomical Pathology, Faculty of Medicine, Cipto Mangunkusumo Hospital by calculating the presentation of $\mathrm{K} 6$ marker expression by immunohistochemical staining. (Figure 2B) Tissue incubation with K6 antibody (LHK6B; Novocastra Laboratories, Newcastle upon Tyne, U.K) for 1 hour with same case without primer antibody as the negative control and skin from breast as the positive control. Two representative sites were selected by an expert in Anatomical Pathology Department and the presentation of K6 marker expression in each location was then calculated and subsequently was summed and the mean value was calculated.

Patients were asked to apply the given topical medication on their skin according to the instruction, i.e. twice daily after having a bath. Patients were asked to keep the ointment on their skin for at least about 30 minutes and after that patients were allowed to do activities such as wearing their trousers, etc. After 4 weeks, the very same evaluation as had been conducted in the beginning of the study was performed again and the results were analyzed by chi-square test using STATA V.12 software.

\section{RESULTS}

\section{Socio-demographic characteristics}

At the end of determined limit of study period, only 17 patients participated in the study. This was due to the decreasing number of patients with mild psoriasis who came to seek treatment. Two subjects were dropped out, one subject could not come for visit due to work hour. One subject became moderate psoriasis, so he needed other treatment that might cause bias in this study.

Of the 17 subjects, there were 2 age groups with the greatest number of subjects, i.e. the age group of 31 to 40 years and 51 to 59 years with 5 subjects $(29.4 \%)$ for each group and most subjects were male (13 subjects or $76.5 \%$ ). Eight subjects (47.1\%) had an education level equal to university level. Fourteen subjects (82.3\%) had been married and 10 subjects (58.8\%) worked as private employees.

Six subjects (35.3\%) suffered from psoriasis for less than 12 months and 5 subjects (29.4\%) had suffered psoriasis for more than 60 months. All subjects had received treatment for psoriasis and two of them had received systemic treatment or phototherapy prior the study. The initial median of PASI in the group of lesions treated with $1 \%$ C. xanthorrhiza ointment was 0.158 ( $\min 0.009$, $\max 1.015$ ); while in the group of lesions treated with placebo, it was 0.157 ( $\mathrm{min}$ 0.13 , max 0.645$)(p=0.561)$. The mean of Trozak score in the group of lesions treated with $1 \% C$. xanthorrhiza ointment was 9.43 (SD 4.29) and in the group of lesion treated with placebo was 6.38 (SD 3.62) $(p=0.058)$. The mean of K6 expression presentation in the group of lesions treated with $1 \%$ topical C. xanthorrhiza was 0.54 (SD 0.13) and in the group of lesions treated with placebo, it was 0.59 (SD 0.16) ( $\mathrm{p}=0.365)$.

PASI, Trozak score, and $\mathrm{K} 6$ expression presentation after $1 \%$ C. xanthorrhiza ointment or placebo treatment

The PASI, Trozak scores, K6 expression presentation prior and after treatment are listed in table 1 and figure 3. 

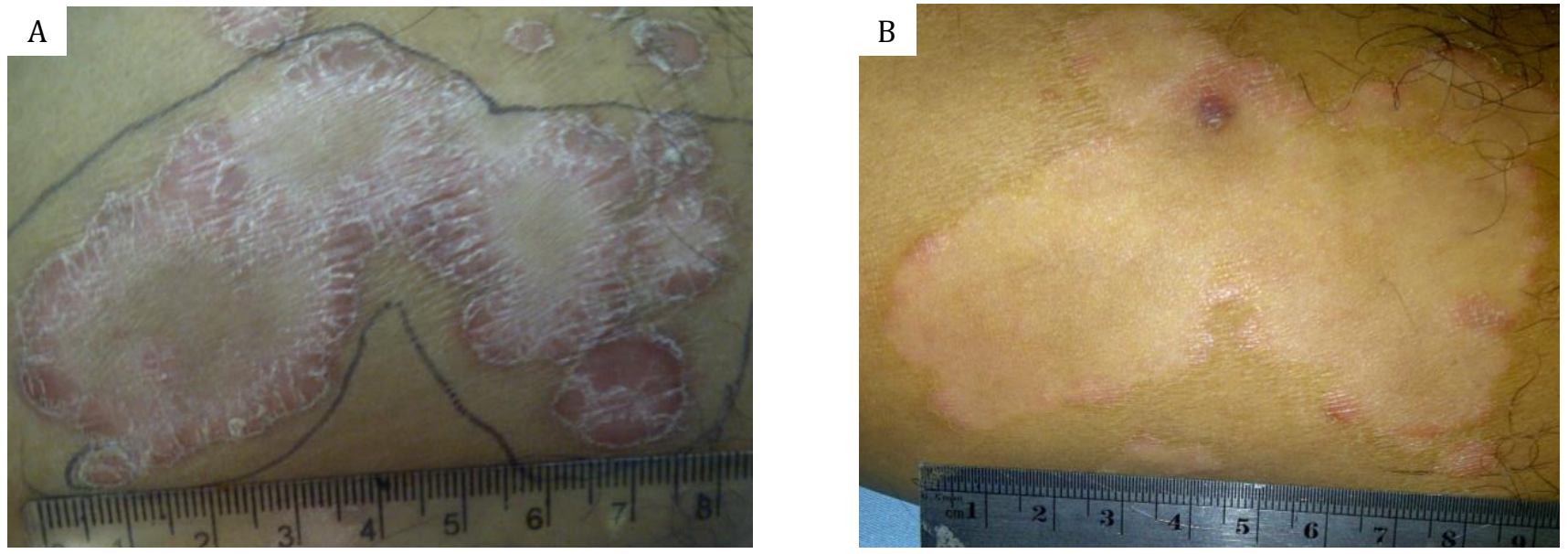

Figure 1. A) and B) PASI score calculation before and after treated with $1 \%$ C. xanthorrhiza ointment. PASI score calculation: the sum of erythema severity (1-4), infiltration (1-4) and scales (1-4), multiply with the percentage of affected body surface area.
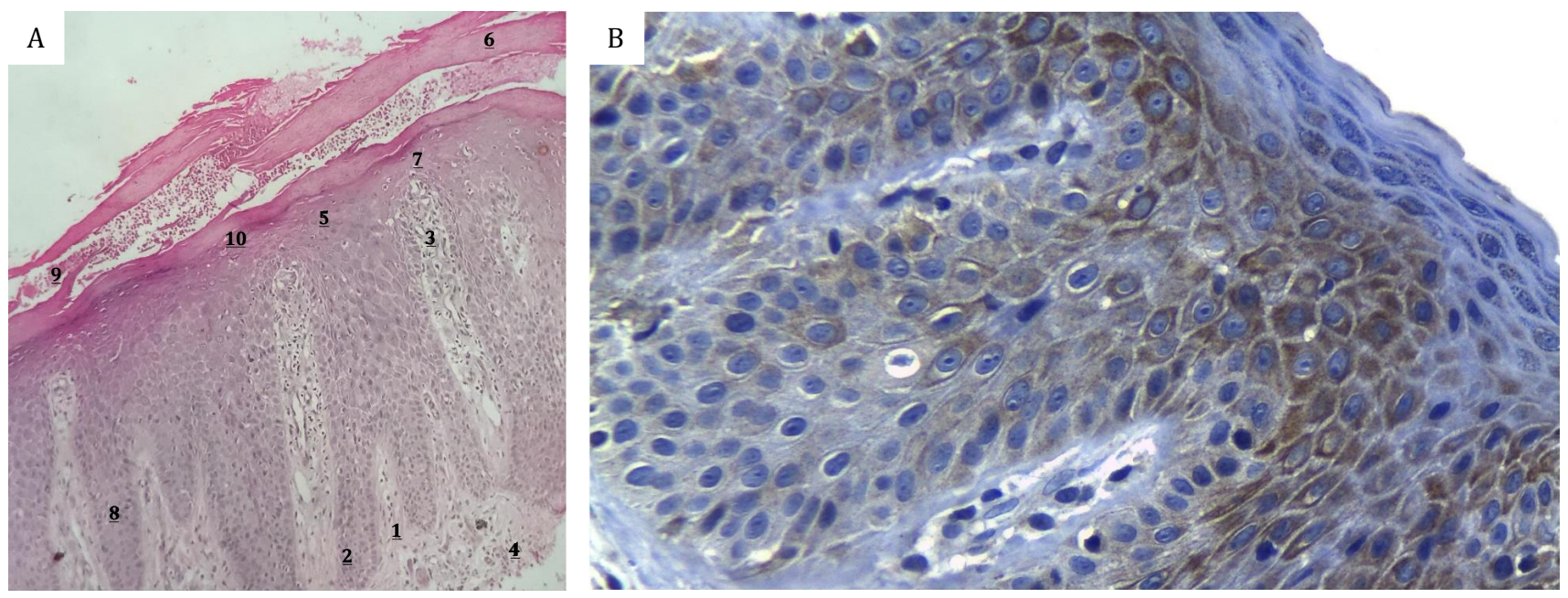

Figure 2. A) Hematoxylin eosin staining for Trozak score calculation of psoriasis lesion; B) Immunohistochemical staining for K6 expression presentation calculation of psoriasis lesion. Trozak score calculation: 1 . regular elongation of the rete ridges (1) 2. club shaped rete ridges (2) 3. elongation and edema of dermal papillae (1) 4. perivascular mononuclear infiltrate in the upper dermis of papillae (1) 5. absence of granular layer (focal:1, total:2) 6. parakeratosis (focal:1, total:2) 7. suprapapillary plate thinning (2) 8. mitosis above basal cell layer (2) 9. Munro microabscess (3) 10. spongiformis pustule (3)

\section{PASI}

In both lesions treated with $1 \% \quad C$. xanthorrhiza ointment and placebo for 4 weeks, there was a significant reduction on median of PASI ( $\mathrm{p}=0.040$ and $\mathrm{p}=0.021$ ). However, when it was compared to those before treatment, the reduction of PASI median or delta $(\Delta)$ between both groups was not significantly different $(\mathrm{p}=0.520)$.

\section{Trozak score}

There was a reduction of Trozak score after $1 \%$ C. xanthorrhiza ointment treatment although the reduction was not statistically significant $(p=0.306)$. In contrast, in the group with placebo treatment, there was a significant increase of Trozak score $(\mathrm{p}=0.039)$. A significant difference was also found in delta $(\Delta)$ of Trozak score between the both groups $(p=0.024)$.

\section{K6 expression presentation}

In group of lesions treated with $C$. xanthorrhiza $1 \%$ ointment, the mean of $\mathrm{K} 6$ expression presentation was $0.54 \quad(\mathrm{SD}=0.13)$ before treatment and $0.55 \quad(\mathrm{SD}=0.17)$ after treatment. There was no significant difference of $\mathrm{K} 6$ expression presentation before and after treated with $1 \%$ C. xanthorrhiza ointment 
Table 1. The difference $(\Delta)$ of PASI score, Trozak score, and K6 expression of psoriasis patients in Cipto Mangunkusumo Hospital before and after $1 \%$ C. xanthorrhiza ointment and placebo treatment

\begin{tabular}{lccc}
\hline \multicolumn{1}{c}{ Variable } & C. xanthorrhiza & Placebo & p value \\
\hline PASI score, median (min-max) & $0.059(-0.03-0.815)$ & $0.047(-0.087-0.613)$ & 0.520 \\
Trozak score, mean (SD) & $1.5(5.27)$ & $-3.45(4.84)$ & $\mathbf{0 . 0 2 4}$ \\
K6 expression, mean (SD) & $0.007(0.14)$ & $0.02(0.20)$ & 0.827 \\
\hline
\end{tabular}

PASI=psoriasis area severity index
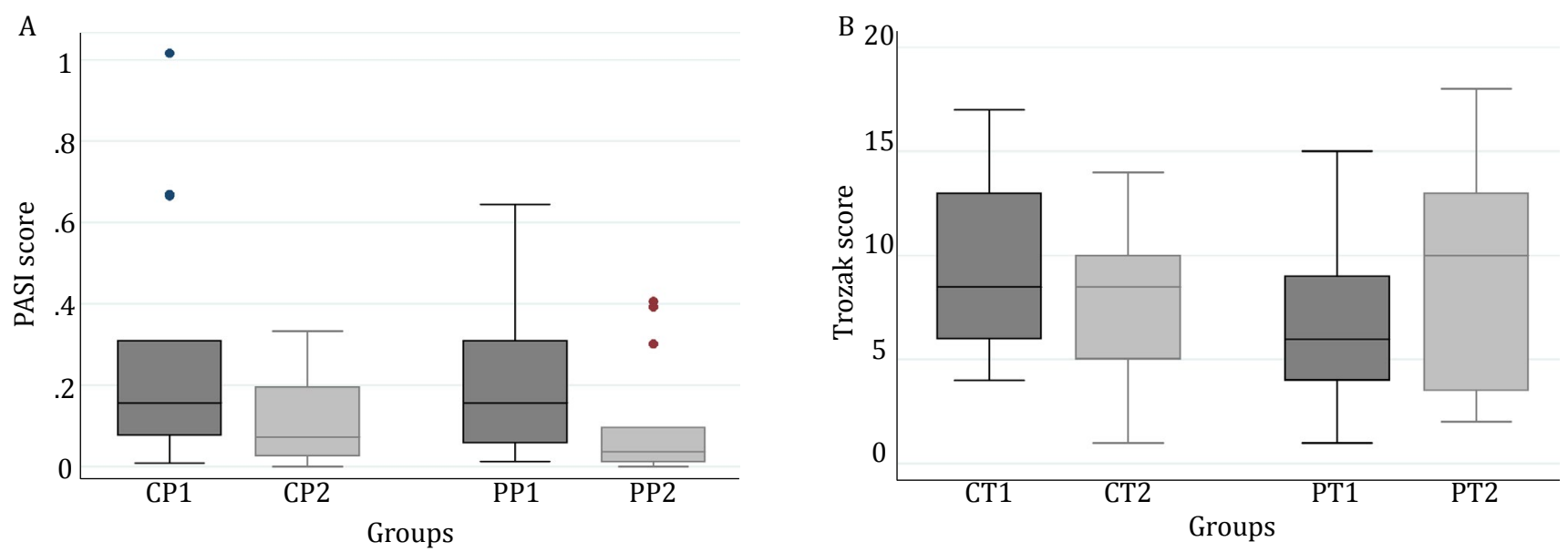

Figure 3. A) PASI score of psoriasis patients in Cipto Mangunkusumo Hospital before $1 \%$ C. xanthorrhiza ointment treatment (CP1), after 1\% C. xanthorrhiza ointment treatment (CP2), before placebo treatment (PP1), and after placebo treatment (PP2); B) Trozak score of psoriasis patients in Cipto Mangunkusumo Hospital before $1 \%$ C. xanthorrhiza ointment treatment (CT1), after $1 \%$ C. xanthorrhiza ointment treatment (CT2), before placebo treatment (PT1), and after placebo treatment (PT2)

$(\mathrm{p}=0.839)$.In group oflesions treated with placebo, the mean of K6 expression presentation was 0.59 $(\mathrm{SD}=0.16)$ before treatment and $0.62(\mathrm{SD}=0.21)$ after treatment. There was no significant difference of $\mathrm{K} 6$ expression presentation before and after treated with placebo $(\mathrm{p}=0.684)$. Similar result was also found in mean difference delta $(\Delta)$ of $\mathrm{K} 6$ expression before and after treatment of $1 \%$ C. xanthorrhiza ointment and placebo $(p=0.827)$. During or after the study has been completed, no subjects has reported any side effects, neither mild nor severe side effects. The $1 \%$ C. xanthorrhiza ointment was well tolerated by all subjects.

\section{DISCUSSION}

The present study was conducted to identify the effectiveness of $1 \%$ C. xanthorrhiza ointment on mild psoriasis compared to placebo treatment for 4 weeks. The parameters that had been evaluated were clinical severity including local PASI, histopathological measure using Trozak score and K6 expression using immunohistochemical staining. About 17 subjects were involved in the study and 2 subjects were dropped out because 1 subject was not able to come for visit and 1 subject became moderate psoriasis. In the present study, no side effect was found, either mild or severe side effects.

For PASI score, the severity of psoriasis was evaluated based on the degree of erythema and induration of plaque thickness, which characterized the presence of inflammation. Moreover, the scales thickness indicates the presence of excessive keratinocytes production. There was a significant reduction of PASI median in the group of lesions treated with $1 \%$ C. xanthorrhiza ointment. Furthermore, in the group with placebo treatment, there was also a significant reduced median of PASI. The reduction of PASI median delta $(\Delta)$ between both group was not significantly different. Reduced PASI in the group of lesions treated with $1 \%$ C. xanthorrhiza 
ointment maybe associated with the antiinflammatory effect of $C$. xanthorrhiza.

Curcuma xanthorrhiza contains the phytochemical component called curcumin. In India, curcuminis usuallyused as a topical ointment for the treatment of inflammation. ${ }^{14}$ Heng et $\mathrm{al}^{12}$ compared topical Curcuma and calcipotriol for psoriasis. He reported that curcumin can lower the activity of phosphorylase kinase enzyme more than calcipotriol. The decrease in phosphorylase kinase activity in curcumin and calcipotrioltreated psoriasis was associated with a decrease in keratinocyte transferrin receptor expression and with decrease in the severity of parakeratosis and the density of epidermal CD8+ T cells. They concluded that drug-induced suppression of phosphorylase kinase activity is associated with resolution of psoriatic activity and that the antipsoriatic activity of curcumin may be achieved through modulation of phosphorylase kinase activity. Sarafian et $\mathrm{al}^{11}$ reported significant reduced PASI and Dermatology Life Quality Index (DLQI) score in 34 patients with mild to moderate plaque psoriasis in a randomized, right-left comparative, placebo-controlled, double-blind clinical trial. The patients were treated with topical turmeric (Curcuma longa) microemulgel, twice a day for 9 weeks. Microemulgel was based on microemulsion, which in previous study had the fastest release through nylon membrane and cellulose membrane compared with gel or ointment. Different result in our study probably due to different vehicle and treatment duration. On the other hand, Kang et $\mathrm{al}^{15}$ concluded that curcumin showed excellent therapeutic effect against psoriasis in mouse model. Similar result was also found in the lesions treated with placebo. The placebo used in the study was vaseline album, which has moisturizing effect. Liu et $\mathrm{al}^{16}$ used a moisturizer containing linoleic acid and ceramide as additional ingredients to $0.1 \%$ momethasone furoate in 106 psoriasis patients. They reported significant improved PASI at the 8th week in the group using moisturizer with less frequent relapse and rebound incidence. They concluded that a moisturizer containing linoleic acid and ceramides can reduce inflammation in psoriasis, which also serves as prevention. Similar to those results, in this study the moisturizing effect of the placebo or vaseline album, which is the vehicle in $1 \%$ C. xanthorrhiza ointment maybe also associated with the reduction of PASI.
The specimens from biopsy were stained with HE and Trozak score was assessed which consists of the presence and absence of elongated rete ridges, the shape of club, elongation and edema of papillary dermis, perivascular infiltrates, the presence or absence of granular layer, parakeratosis, thinning of suprapapillary layer, Munro abscess and spongiformis. A decreasing of Trozak score was found after 4-week treatment using $1 \%$ C. xanthorrhiza ointment. However, the reduction was not statistically significant. While in the group treated with placebo, there was a significant increase of Trozak score. The difference or delta $(\Delta)$ between both groups was also significantly different. Reduced Trozak score in this study maybe caused by the anti-inflammatory effect of $C$. xanthorrhiza. In his report, Trozak DJ ${ }^{13}$ has developed an assessment system to obtain objective results of the study on drug effectiveness.

In clinical manifestation(PASI score), $1 \% C$. xanthorrhiza ointment and placebo which contain vaseline album reduced inflammation in patients with mild psoriasis. However, in histopathological features (Trozak score), the effectivity of vaseline album to reduce inflammation is less than $1 \%$ C. xanthorrhiza ointment. The histopathological examination of the skin that appears normal in psoriasis patients revealed that there is already a mild capillary and curvature dilatation and a slight increasing in the number of mononuclear cells of the dermis and mast cells. Moreover, the study also found that there was a slight increasing in epidermal thickness. ${ }^{1}$ These results showed that although psoriasis lesion has not been appeared clinically, some changes in histopathology have already occurred. This condition can explain the significant increase of Trozak score and the significant reduction of PASI score in the placebo group from this study. If longer term of follow up was carried out, PASI score might be increased.

K6 is a marker of differentiation disorder and it is expressed by activated keratinocytes, particularly in inflammation. ${ }^{1}$ The expression of K6 in keratinocytes can be identified using immunohistochemical staining. There was no significant difference of K6 expression presentation before and after treatment, either in thegroup oflesionstreated with $1 \%$ C.xanthorrhiza ointment or placebo. The difference of the delta $(\Delta)$ of $\mathrm{K} 6$ expression before and after treatment of $1 \%$ C. xanthorrhiza ointment and placebo 
was also not significant. Körver et al ${ }^{17}$ compared the effectiveness of calcitriol and calcipotriol ointment in psoriasis for 6 weeks. They wanted to identify the effect of those topical treatments on the epidermal cell population, proliferation and differentiation. They reported reduced expression of $\mathrm{K} 6$ with immunohistochemical staining in the group treated with calcipotriol ointment. In this study, there was no significant difference of $\mathrm{K} 6$ expression either on the group with $1 \%$ C. xanthorrhiza ointment or placebo. It might be due to shorter treatment duration than those in the literature ( 4 weeks).

In this study, there was no side effect reported, either mild or severe. On the other hand, Sarafian et al ${ }^{11}$ in their study using turmeric (Curcuma longa) microemulgel for 34 psoriasis patients compare to placebo, reported 6\% patients experienced dryness and 3\% patients experienced irritation. They concluded it could be associated with the inactive ingredients of the formulation than the turmeric itself. In this study, we used vaseline album as the vehicle, this might cause the different result.

In conclusion, the $1 \%$ C. xanthorrhiza ointment appears to be an effective alternative treatment option for mild psoriasis based on significant reduction of PASI statistically and Trozak score. It may be interpreted that $C$. xanthorrhiza can at least, prevent the worsening of disease severity. Further study with longer treatment duration and follow up period is recommended to confirm these results.

\section{Conflict of Interest}

This study was funded by PT. Soho Industri Pharmasi for topical formulations and in cooperation with Indonesian Psoriasis Study Group Indonesian Society of Dermatology and Venereology.

\section{Acknowledgment}

The authors thank the patients for their participation in this study. Special gratitude is also given to Yayasan Peduli Psoriasis Indonesia who had participated in referring their members as patients in this study. The authors would like to thank Dr. dr. Nurjati Siregar, SpPA from Anatomical Pathology Department of Cipto Mangunkusumo Hospital in processing immunohistochemical staining and K6 expression counting.

\section{REFERENCES}

1. Gudjonsson JE, Elder JT. Psoriasis. In: Wolf K, Goldsmith LA, Katz SI, Gilchrest BA, Paller AS, Leffel DJ, editors. Fitzpatrick's Dermatology in General Medicine. $8^{\text {th }}$ Edition. New York: McGraw-Hill; 2012. p.197-231

2. Grove T. The pathogenesis of psoriasis: Biochemical aspects. [cited February 25, 2007]. Available from: http://www.jyi.org/issue-4/groove.html

3. Ghosh A, Panda S. Recent understanding of the etiopathogenesis of psoriasis. Indian J Paediatr Dermatol. 2017;18(1):1-8.

4. Capon F. The genetic basis of psoriasis. Int J Mol Sci. 2017;18(2):2526.

5. Mustafa AA, Al-Hoqail IA. Biologic systemic therapy for moderate-to-severe psoriasis: a review. J Taibah Univ Sci. 2013;8(3):142-50.

6. Menter A. Recent advances in psoriasis therapy and the work of international psoriasis council. US Derm Rev. 2006;1:23-7.

7. Sastroamidjoyo S. Daftar tumbuh-tumbuhan yang dapat dipergunakan sebagai bahan-bahan obat asli Indonesia. In: Tjokronegoro A, editor. Obat asli Indonesia. Jakarta: Dian Rakyat;1997. p.254-5

8. Maria Laksmi Parahita. Curcuma xanthorrhiza (temulawak) morfologi, anatomi, dan fisiologi. [cited 20 Februari 2007]. Available from: http://toiusd.multiply. com/journal/item/240.

9. Suyono Hadi. Manfaat temulawak ditinjau dari segi kedokteran. Naskah lengkap Simposium Nasional Temulawak, Bandung: UNPAD, 1985.

10. Miquel J, Bernd A, Sempere JM, Díaz-Alperi J, Ramírez A. The curcuma antioxidants: pharmacological effects and prospects for future clinical use. a review. Arch Gerontol Geriatr. 2002;34(1):37-46.

11. Sarafian G, Afshar M, Mansouri P, Asgarpanah J, Raoufinejad K, Rajabi M. Topical turmeric microemulgel in the management of plaque psoriasis; a clinical evaluation. Iran J Pharm Res. 2015;14(3):865-76.

12. Heng MC, Song MK, Harker J, Heng MK. Drug- induced suppression of phosphorylase kinase activity correlates with resolution of psoriasis as assessed by clinical, histological and immunohistochemical parameters. Br J Dermatol. 2000;143(5):937-49.

13. Trozak DJ. Histologic grading system for psoriasis vulgaris. Int J Dermatol. 1994;33(5):380-1.

14. Jacob A, Wu R, Zhou M, Wang P. Mechanism of the antiinflammatory effect of curcumin: PPAR- $\gamma$ activation. PPAR Res. 2007;2007:89369.

15. Kang D, Li B, Luo L, Jiang W, Lu Q, Rong M' Lai R. Curcumin shows excellent therapeutic effect on psoriasis in mouse model. Biochimie.2016 Apr;123:73-80.

16. Liu M, Li X, Chen XY, Xue F, Zheng J. Topical application of linoleic acid-ceramide containing moisturizer exhibit therapeutic and preventive benefits for psoriasis vulgaris: a randomized controlled trial. Dermatol Ther. 2015;28(6):373-82.

17. Körver JE, Visser WH, van Rens DW, Pasch MC, van Erp PE, BoezemanJB, et al. Adouble blind, randomized quantitative comparison of calcitriol ointment and calcipotriol ointment on epidermal cell population, proliferation and differentiation. Br J Dermatol. 2007;156(1):130-7. 